\title{
Thrombopoietin Promotes Angiogenesis and Disease Progression in Patients with Multiple Myeloma
}

Aurelia Lamanuzzi, ${ }^{*}$ Ilaria Saltarella, ${ }^{*}$ Maria A. Frassanito, ${ }^{\dagger}$ Domenico Ribatti, ${ }^{\ddagger}$ Assunta Melaccio, ${ }^{*}$ Vanessa Desantis, ${ }^{*}$ Antonio G. Solimando, ${ }^{\star}$ Roberto Ria, ${ }^{*}$ and Angelo Vacca*

From the Department of Biomedical Sciences and Human Oncology, * Unit of Internal Medicine and Clinical Oncology, the Department of Biomedical Sciences and Human Oncology, ${ }^{\dagger}$ Unit of General Pathology, and the Department of Basic Medical Sciences, Neurosciences and Sensory Organs, ${ }^{\ddagger}$ University of Bari Aldo Moro, Bari; and the Medical Oncology Unit, ${ }^{\S}$ IRCCS Istituto Tumori "Giovanni Paolo II" of Bari, Bari, Italy

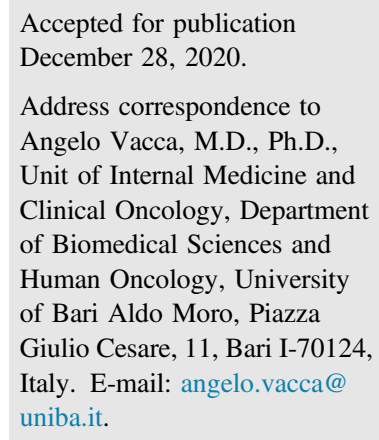

\begin{abstract}
Multiple myeloma (MM) progression closely depends on bone marrow (BM) angiogenesis. Several factors sustain angiogenesis, including cytokines, growth factors, and cell-to-cell interactions. Herein, BM thrombopoietin (TPO) was shown to support angiogenesis and disease progression in MM. Patients with MM at different progression phases had higher levels of BM and circulating TPO than monoclonal gammopathy of undetermined significance/smoldering MM patients, suggesting that TPO correlates with disease progression and prognosis. Endothelial cells from patients with monoclonal gammopathy of undetermined significance (MGECS) and endothelial cells from MM (MMECs) expressed TPO receptor, and the TPO treatment triggered their angiogenic capabilities in vitro. Indeed, TPO-treated MGECS and MMECs showed enhanced angiogenesis on Matrigel and spontaneous cell migration and chemotaxis by acting as a chemotactic agent. TPO also had an angiogenic activity in vivo in the chorioallantoic membrane assay system. Finally, TPO treatment increased the release of active matrix metalloproteinase (MMP)-9 and MMP-2 in MGECs and of MMP-2 in MMECs and affected the balance between angiogenic/ antiangiogenic factors in the MM BM. Our results support the angiogenic activity of TPO, and suggest that it may have a critical role in promoting the angiogenic switch during MM progression. Accordingly, TPO may be envisaged as a new angiogenic and prognostic factor in patients with MM. (Am J Pathol 2021, 191: 748-758; https://doi.org/10.1016/j.ajpath.2020.12.016)
\end{abstract}

Multiple myeloma (MM) is characterized by the monoclonal expansion and accumulation of Ig-secreting plasma cells within the bone marrow (BM). ${ }^{1}$ The BM microenvironment plays an essential role in plasma cell proliferation, angiogenesis, invasion, and metastasis, providing survival signals, growth, and angiogenic factors. ${ }^{2}$ The complex interplay between tumor and stromal cells modifies the BM microenvironment and contributes to the angiogenic switch from the avascular phase of monoclonal gammopathy of undetermined significance (MGUS) to the vascular phase of MM via autocrine and paracrine loops. ${ }^{3}$ Angiogenesis is a physiological and pathologic multistep process consisting of new vessel formation from preexistent adjacent ones. It is an essential hallmark of MM progression and correlates with tumor growth, relapse, and drug resistance. ${ }^{3}$ The novel drugs in MM management (ie, the proteasome inhibitors and the immunomodulatory drugs) have both antiangiogenic and cytotoxic effects. ${ }^{4,5}$ Moreover, the introduction of anti-vascular endothelial

Supported by INNOLABS-Programma Operativo Regionale (POR) Puglia Fondo Europeo di Sviluppo Regionale (FESR)-Fondo Sociale Europeo (FSE) 2014 to 2020 Telemielomedicina/Telemielolab (A.V.) and by Apulian Regional project: Precision Medicine number 06062019 (A.G.S.).

The sponsors of this study are public or non-profit organizations that support science in general.

A.L. and I.S. contributed equally to this work.

R.R. and A.V. contributed equally to this work as senior authors.

Disclosures: None declared. 
growth factor (VEGF) monoclonal antibodies, histone deacetylase inhibitors, and tyrosine kinases inhibitors, such as bevacizumab, panobinostat, SU11274, sunitinib, and sorafenib, ${ }^{6,7}$ has shown high anti-MM and antiangiogenic effectiveness in in vitro systems, and a significant but not enduring therapeutic benefit in patients. Conventional and high-dose chemotherapies induce treatment-related adverse events (ie, thrombocytopenia) ${ }^{8}$; furthermore, MM plasma cell infiltration in BM prompts thrombocytopenia by triggering thrombopoietin (TPO) production. ${ }^{9}$

TPO is the primary regulator of the proliferation and maturation of megakaryocytes as well as of platelet production. ${ }^{10}$ It is primarily synthesized in the liver, kidney, ${ }^{11}$ and $\mathrm{BM},{ }^{12}$ but the mechanism by which TPO is produced and secreted by BM is not yet clear. ${ }^{12}$ TPO receptor (c-Mpl; $\mathrm{CD} 110$ ) is a member of the type I cytokine receptor family, and it is expressed on several hematopoietic cells (ie, stem cells, megakaryocyte colony-forming cells, myeloid and erythroid progenitors, early and late megakaryocytes, and mature platelets). Brizzi et $\mathrm{al}^{13}$ demonstrated that the human umbilical vein endothelial cells (HUVECs) express functional TPO receptor whose activation promotes in vitro and in vivo angiogenesis. The c-Mpl is expressed as a preformed, but inactive, dimer. After TPO binding, c-Mpl is internalized and activates several signal transduction pathways: the Janus kinase/STAT pathway, which promotes cell proliferation; the rat sarcoma/rapidly accelerated fibrosarcoma (RAS/RAF) and mitogen-activated protein kinase pathways, which induce cell differentiation and maturation; and the Akt pathway, which is involved in apoptosis resistance. $^{14}$

Despite the development of new drugs, MM is still an incurable disease, and the discovery of new targets and the development of new biologic drugs is warranted. The failure of MM treatment is also caused by a vivid angiogenesis that sustains MM progression and, finally, leads to drug resistance.

Herein, we demonstrate that TPO has an angiogenic effect on BM-derived ECs (BMECs) and that BM/peripheral TPO plasma levels correlate with MM progression. On the basis of these observations, we speculate that TPO may be envisaged as a new angiogenic and prognostic factor in patients with MM.

\section{Materials and Methods}

\section{Patients}

Patients fulfilling the International Myeloma Working Group diagnostic criteria (IMWG2014 $\left.{ }^{15}\right)$ for MM $(n=55)$ and MGUS $(n=16)$ /smoldering MM (SMM; $n=4)$ were studied (Table 1).

The study was approved by the Ethics Committee of the University of Bari Aldo Moro Medical School (protocol number 0086738|07/11/2017), and patients provided their
Table 1 General Characteristics and Diagnostic Features of the Patients Studied

\begin{tabular}{lll}
\hline Variable & MGUS/SMM & MM \\
\hline Patients, $n$ & $16 / 4$ & 55 \\
Age, median (range), years & $54(36-86)$ & $64(41-89)$ \\
Sex, $n$ /total & & \\
$\quad$ Male & $9 / 20$ & $29 / 55$ \\
$\quad$ Female & $11 / 20$ & $26 / 55$ \\
ISS, $n$ /total & & \\
Stage I & & $16 / 55$ \\
Stage II & & $22 / 55$ \\
$\quad$ Stage III & & $17 / 55$ \\
Type of MM, $n /$ total & $16 / 20$ & \\
IgG & & $39 / 55$ \\
IgA & & $12 / 22$ \\
IgD & & $1 / 55$ \\
Light chain & $4 / 20$ & $2 / 55$ \\
$\quad$ Not secreting & & $1 / 55$ \\
Median platelet count, cells/mL & \\
Stage I & 232,000 & 190,000 \\
Stage II & & 230,000 \\
Stage III & & 198,000 \\
Median plasma cells, \% & \\
Stage I & 3 & 14.5 \\
Stage II & & 30 \\
Stage III & & 65 \\
\hline
\end{tabular}

ISS, International Staging System; MGUS, monoclonal gammopathy of undetermined significance; MM, multiple myeloma; SMM, smoldering MM.

informed consent in accordance with the Declaration of Helsinki.

\section{EC Isolation and Characterization}

BM mononuclear cells from MM and MGUS patients were obtained by centrifugation on Ficoll-Hypaque (Pharmacia Biotech, Uppsala, Sweden) gradient of heparinized BM aspirates. Cells were cultured in RPMI 1640 medium supplemented with $10 \%$ fetal bovine serum (FBS) and $1 \%$ antibiotic/antimycotic. To isolate ECs, BM adherent stromal cells were harvested and incubated with magnetic microbeads coated with anti-CD31 antibody (Miltenyi Biotec, Bergisch Gladbach, Germany), according to manufacturer's instructions. Factor VIII-related antigen, CD31, vascular endothelial growth factor receptor 2 , and tyrosine kinase with immunoglobulin-like and EGF-like domains 2 (Tie2) expression levels on freshly isolated endothelial cells from MGUS (MGECs) and endothelial cells from MM (MMECs) patients were analyzed by flow cytometry, FACS Canto II (Becton Dickinson, San Jose, CA).

\section{Reagents}

Recombinant human thrombopoietin, Dulbecco's modified Eagle's medium (DMEM), RPMI 1640 medium, heatinactivated FBS, antibiotic/antimycotic, trypsin/EDTA, 
and phosphate-buffered saline without $\mathrm{Ca}^{+}$and $\mathrm{Mg}^{+}$ were obtained from Sigma-Aldrich (St. Louis, MO).

\section{TPO Detection}

TPO levels were analyzed on paired BM/peripheral plasma of patients with MGUS and MM at different progression phases using Human Thrombopoietin Quantikine ELISA (R\&D Systems, Inc., Minneapolis, MN), according to the manufacturer's instructions. TPO levels were measured and quantified with VICTOR X3 Multilabel Plate Reader (PerkinElmer Inc., Waltham, MA).

\section{Flow Cytometry}

Expression of surface human thrombopoietin receptor (c-Mpl; CD110) on BMECs was detected using a specific monoclonal antibody (Becton Dickinson; CD110), according to the manufacturer's instructions. Samples were acquired to flow cytometry (FACScanto II; Becton Dickinson) and analyzed using FACS Diva software version 6.1.2 (Becton Dickinson).

\section{Immunoprecipitation and Western Blot Analysis}

Total protein lysate $(600 \mu \mathrm{g})$ from BMECs was immunoprecipitated using anti-c-Mpl polyclonal antibody (Santa Cruz Biotechnology, Dallas, TX) with Immunoprecipitation Dynabeads Protein G Kit (Invitrogen Corp., Carlsbad, CA), according to the manufacturer's instructions. Next, samples were analyzed by Western blot analysis: they were separated on $4 \%$ to $12 \%$ NuPAGE gels (Invitrogen Corp.), electrotransferred to a polyvinylidene difluoride membrane (PerkinElmer Life Science Inc., Boston, MA), and immunoblotted with anti-c-Mpl monoclonal antibody (Santa Cruz Biotechnology). $\beta$-Actin was used as loading control, and analyzed on total lysate using anti- $\beta$-actin antibody (Sigma-Aldrich). Then, the membranes were incubated with horseradish peroxidase-labeled secondary antibodies (Bio-Rad, Hercules, CA). Immunoreactive bands were visualized by enhanced chemiluminescence (LiteAblot extend substrate; Euroclone, Pero, Italy) and the Gel Logic 1500 Imaging System (Eastman Kodak Co, Rochester, NY).

\section{Viability Assay}

BMECs were seeded at 1000 cells per well in 96-well plates in DMEM supplemented with 20\% FBS and $1 \%$ antibiotic/ antimycotic (complete medium). After 12 hours, the medium was removed, and cells were washed twice with phosphate-buffered saline. Then, cells were cultured in DMEM supplemented with TPO at different concentration $(1 \div 1000 \mathrm{ng} / \mathrm{mL})$. Cell viability was evaluated with CellTiter-Glo Luminescent Cell Viability Assay (Promega Corp., Madison, WI), according to the manufacturer's instructions. Luminescence was quantified with VICTOR X3 Multilabel Plate Reader (PerkinElmer Inc.) in sextuplicate.

\section{Proliferation Assay}

BMECs $\left(1 \times 10^{5}\right)$ were labeled with viable probe carboxyfluorescein succinimidyl ester (Invitrogen Corp.), according to the manufacturer's instructions. Cells were cultured in serum-free medium (SFM) or DMEM supplemented with $10 \mathrm{ng} / \mathrm{mL}$ TPO for 72 hours. Samples were acquired to the FACScanto II flow cytometer, and analyzed using FACS Diva software.

\section{Apoptosis Assay}

BMECs $\left(1 \times 10^{5}\right)$ were cultured in SFM or DMEM supplemented with $10 \mathrm{ng} / \mathrm{mL}$ TPO for 72 hours. Apoptosis was assessed by Annexin-V-PE/7-AAD (Becton Dickinson) staining and flow cytometry analysis, according to manufacturer's instructions.

\section{Angiogenesis Assay on Matrigel}

BMECs were plated $\left(2 \times 10^{4}\right)$ on 48 -well plates coated with Matrigel (Becton Dickinson) in SFM, as control, and in DMEM supplemented with TPO $(10 \mathrm{ng} / \mathrm{mL})$. After 16 hours, the skeletonization of the mesh was followed by measurement of mesh areas, vessel length, and branching points in three randomly chosen fields with the EVOS inverted microscope (Euroclone) at $\times 10$ magnification.

\section{Wound-Healing Assay}

BMECs $\left(4 \times 10^{4}\right)$ were seeded on 24 -well plates in complete medium. The day after a wound was made by scraping the cell monolayer with a sterile pipette tip, medium was removed, and the cells were washed with phosphatebuffered saline. Then, cells were cultured in SFM (as negative control), DMEM with $1.5 \%$ FBS added with VEGF and fibroblast growth factor-2 (both $10 \mathrm{ng} / \mathrm{mL}$; Miltenyi Biotec) as positive control, and TPO at $10 \mathrm{ng} / \mathrm{mL}$. After 16 hours, cells were fixed and stained. Cell migration was determined by counting the cells migrated into the wound in at least three randomly chosen $\times 10$ fields on the EVOS microscope, and indicated as the percentage of relative wound closure compared with the positive control.

\section{Chemotaxis Assay}

The TPO chemotactic activity on BMECs was analyzed on cells $\left(5 \times 10^{4}\right)$ in a Boyden chamber on a polycarbonate membrane (Neuro Probe, Inc., Warwickshire, UK) precoated with $10 \mu \mathrm{g} / \mathrm{mL}$ fibronectin (Sigma-Aldrich). BMEC chemotaxis was tested toward SFM (as negative control), DMEM with $1.5 \%$ FBS added with VEGF and FGF-2 (both 
$10 \mathrm{ng} / \mathrm{mL}$; Miltenyi Biotec; as positive control), and DMEM with TPO at $10 \mathrm{ng} / \mathrm{mL}$. After 16 hours at $37^{\circ} \mathrm{C}$, the migrated cells were fixed, stained, and counted in at least three randomly chosen fields by the EVOS inverted microscope at $\times 40$ magnification.

\section{In Vivo CAM Assay}

Fertilized white Leghorn chicken eggs were incubated at $37^{\circ} \mathrm{C}$ and constant humidity. On third day, the shell was opened, and 2 to $3 \mathrm{~mL}$ of albumen was removed to detach the chorioallantoic membrane (CAM). On eighth day, the CAMs were implanted with $1-\mathrm{mm}^{3}$ sterilized gelatin sponges (Gelfoam Upjohn Co, New York, NY), loaded with SFM alone and TPO at $100 \mathrm{ng} / \mathrm{mL}$. On 12th day, the angiogenic response was evaluated as the number of vessels converging toward the sponge and imaged at $\times 50$ magnification in ovo by a stereomicroscope (Olympus Italia Srl, Segrate, Milan, Italy).

\section{Zymography}

Conditioned media from $2 \times 10^{5}$ BMECs, treated or not with TPO at $10 \mathrm{ng} / \mathrm{mL}$ for 16 hours, were collected and mixed with SDS buffer under nonreducing conditions, and run on Novex Zymogram gelatin gel (Invitrogen Corp.) at $125 \mathrm{~V}$ for 90 minutes. After electrophoresis, the enzyme was renatured by incubating the gel in Zymogram renaturing buffer containing a nonionic detergent. The gel was equilibrated in Zymogram developing buffer, and then stained and destained following manufacturer's instructions (Invitrogen Corp.). Regions of protease activity appeared as clear bands against a dark blue background.

\section{Measurement of Secreted Cytokines}

Conditioned media were obtained from BMECs $\left(2 \times 10^{5}\right)$ cultured in absence and/or presence TPO (10 ng/mL) for 16 hours. Cytokine levels were measured by using Human Angiogenesis Array (R\&D Systems, Inc.) that detects 55 cytokines involved in angiogenesis, according to the manufacturer's instructions. Secreted levels of cytokines were quantified with Kodak Molecular Imaging Software version 2.0.0 (Eastman Kodak Co., Rochester, NY).

\section{Statistical Analysis}

Analyses were performed using GraphPad Prism5 software (GraphPad Software, San Diego, CA). Results were analyzed using the Wilcoxon signed-rank test. $P<0.05$ was considered statistically significant. Results about the correlation between TPO level and platelet count were analyzed using the Pearson correlation coefficient, excluding values up to the 75 degree percentile and down to the 25 degree percentile of TPO concentration. $P<0.005$ was considered statistically significant.

\section{Results}

TP0 Plasma Levels Correlate with MM Progression Phases

To verify the role of TPO in MM progression, its levels were evaluated in BM and peripheral plasma of patients with MGUS and MM at several progression phases. Analysis of BM TPO revealed that MM samples showed higher levels of circulating TPO [SMM, $84.82 \mathrm{pg} / \mathrm{mL}$; first diagnosed (FIR) MM, $154.52 \mathrm{pg} / \mathrm{mL}$; relapsed MM, $450.57 \mathrm{pg} /$ $\mathrm{mL}$; double drug-resistant (DR) MM, $983.63 \mathrm{pg} / \mathrm{mL}$ ] than MGUS samples (27.13 pg/mL) (Figure 1A). Interestingly, BM TPO levels paralleled the MM progression phases. Similar results were obtained by analyzing paired peripheral plasma: MGUS, $52.97 \mathrm{pg} / \mathrm{mL}$; SMM, $75.32 \mathrm{pg} / \mathrm{mL}$; FIR MM, $112.23 \mathrm{pg} / \mathrm{mL}$; relapsed MM, $193.34 \mathrm{pg} / \mathrm{mL}$; and DR MM, $715.23 \mathrm{pg} / \mathrm{mL}$ (Figure 1B), indicating that TPO levels overlap in BM and peripheral samples.
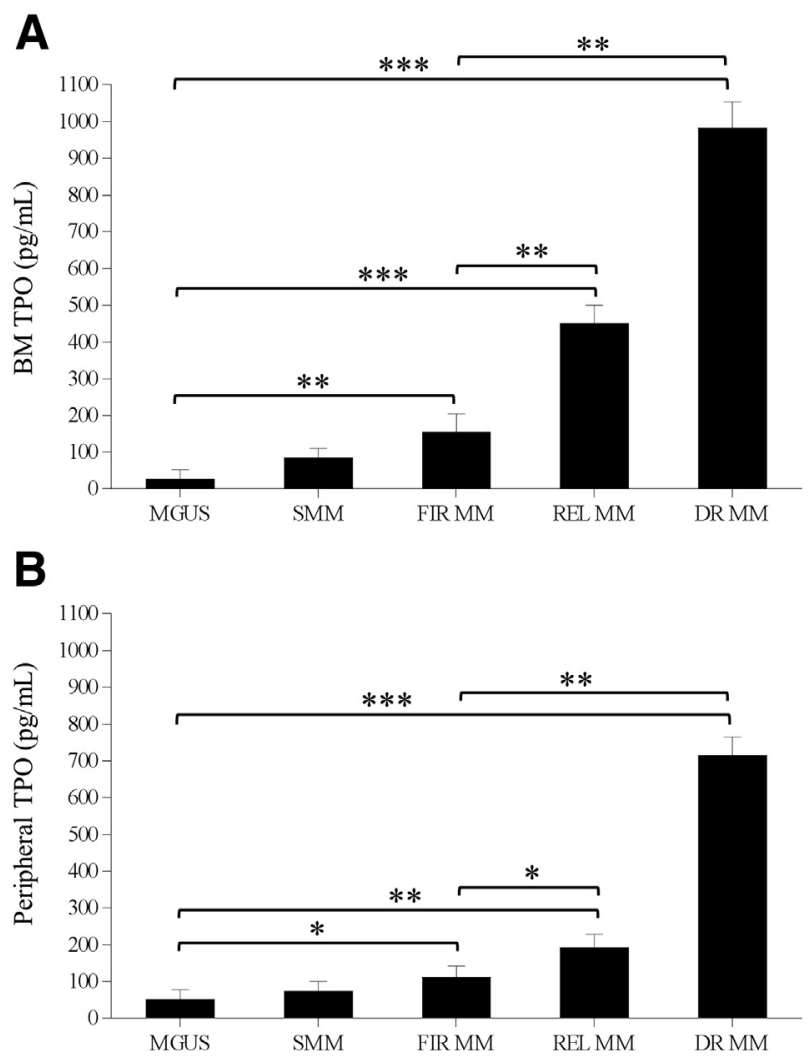

Figure 1 Thrombopoietin (TP0) plasma levels correlate with multiple myeloma (MM) progression phases. Analysis of circulating bone marrow (BM; A) and peripheral TPO (B) in plasma of monoclonal gammopathy of undetermined significance (MGUS), smoldering MM (SMM), first diagnosed (FIR) MM, relapsed (REL) MM, and double drug-resistant (DR) MM patients. Note that TPO circulating levels increase according to the disease progression phases. Data are expressed as means \pm SD (A and $\mathbf{B}$ ). $n=35$ MGUS patients ( $\mathbf{A}$ and $\mathbf{B}$ ); $n=10$ SMM patients; $n=29$ FIR MM patients (A and $\mathbf{B}) ; n=32$ REL MM patients (A and $\mathbf{B}) ; n=13$ DR MM patients (A and $\mathbf{B}) .{ }^{*} P<0.05,{ }^{*} P<0.01$, and ${ }^{* *} * P<0.001$. 


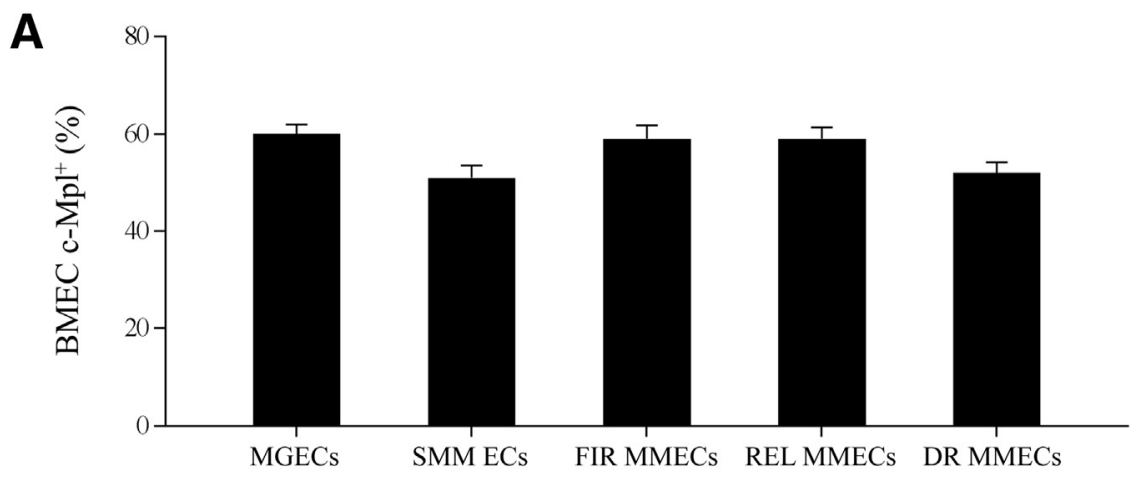

B

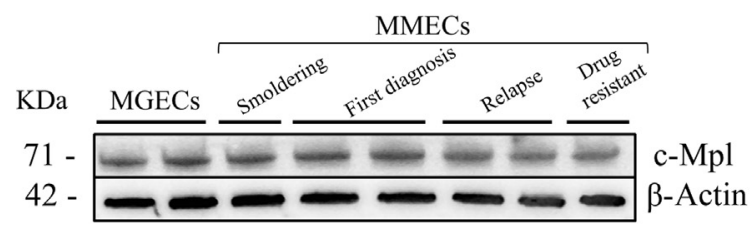

Figure 2 Thrombopoietin receptor (c-Mpl) expression on bone marrow-derived endothelial cells (BMECs). Flow cytometry (A) and Western blot analysis (B) of C-Mpl expression (CD110) on endothelial cells (ECS) from monoclonal gammopathy of undetermined significance (MGUS; MGECS), smoldering multiple myeloma (SMM ECS), first diagnosed (FIR) multiple myeloma (MMECS), relapsed (REL) MMECs, and drug-resistant (DR) MMECs. $\beta$-Actin was used as loading control. Note that all BMECs display a similar c-Mpl expression. Data are expressed as means \pm SD (A). $n=35$ $\operatorname{MGECS}(\mathbf{A}) ; n=10$ SMM ECs (A); $n=29$ FIR MMECS (A); $n=32$ REL MMECS (A); $n=13$ DR MMECS (A). MMECs, EC from MM.
As TPO modulates platelets production, ${ }^{10}$ a link between BM TPO level and platelet count was next analyzed by using the Pearson correlation coefficient. Supplemental Figure S1 shows the negative correlation between TPO level and platelet count. Indeed, platelet count was higher in MGUS and SMM patients with lower TPO levels, whereas increased TPO levels paralleled decreased platelet counts during the worsening of disease $(r=-0.2754 ; P<0.005)$. These data were confirmed in few patients who progressed from MGUS to MM (Patient 1) or FIR to REL/DR MM (Patients 2 and 3), as shown in Supplemental Figure S2.

TPO increased during MM progression; hence, it may correlate with disease prognosis.

\section{BMECs Express TPO Receptor}

To evaluate the role of TPO in MM angiogenesis, first the expression of the TPO receptor c-Mpl (CD110) on BMECs was analyzed. Flow cytometry analysis demonstrated a similar c-Mpl expression in BMECs from patients at different progression phases. Indeed, no significant differences in c-Mpl expression among MGECs (60\%) and MMECs at different phases (SMM, 51\%; FIR MM, 59\%; relapsed MM, 59\%; and DR MM, 52\%) were observed (Figure 2A). These data were confirmed by Western blot analysis of immune-precipitated c-Mpl (Figure 2B).

\section{In Vitro and in Vivo Angiogenic Effects of TPO}

To evaluate the angiogenic effect of TPO, BMECs were treated with TPO and their angiogenic capabilities were tested in vitro. Preliminary dose-finding experiments by treating BMECs with different TPO doses showed that TPO (1 ng/ $\mathrm{mL} \div 10 \mathrm{ng} / \mathrm{mL}$ ) did not affect BMECs viability (Supplemental Figure S3A). Increasing TPO levels (50 ng/
$\mathrm{mL} \div 1 \mu \mathrm{g} / \mathrm{mL}$ ) lightly, but not significantly, reduced BMECs viability (Supplemental Figure S3A). Furthermore, TPO $(5 \mathrm{ng} / \mathrm{mL} \div 30 \mathrm{ng} / \mathrm{mL})$ induced a dose-dependent angiogenic effect on MMECs by enhancing angiogenesis on Matrigel, whereas higher TPO concentrations did not further increase the intrinsic MMECs angiogenic ability (Supplemental Figure S3B). On the basis of these preliminary results, BMECs from patients at different progression phases were treated with TPO at $10 \mathrm{ng} / \mathrm{mL}$. No effect on cell proliferation and apoptosis was observed (Supplemental Figure S4).

MGECs have low angiogenic ability on Matrigel (Figure 3A). TPO treatment increased the formation of branching points, anastomosing tubes with multicentric junctions along with the origin of a meshwork of capillary-like structures (Figure 3, B-D). Although MMECs of patients with FIR MM had intrinsic angiogenic ability in vitro (Figure 3, E-H) ${ }^{16}$ the TPO exposure gave their overangiogenic phenotype because it further improved the MMECs angiogenic network, by increasing the density of branching points (Figure $3 \mathrm{H}$ ). Similar results were obtained by treating MMECs from patients with $\mathrm{MM}$ at different progression phases (data not shown).

As angiogenesis involves different functionalities of ECs the TPO capability to improve the angiogenesis-related functions was investigated. As shown in Figure 4, A-D, TPO treatment increased spontaneous migration in MGECs and MMECs: the TPO-treated BMECs filled the wound much in the same way as the positive control. Next, the TPO chemoattractant ability was evaluated by performing a chemotherapy-induced migration assay with the Boyden chamber. Interestingly, TPO significantly raised MGECs and MMECs chemotaxis, suggesting that it may act as a chemotactic factor (Figure 4, E-H). 


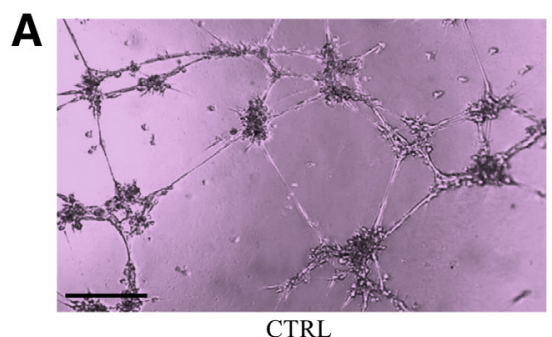

B
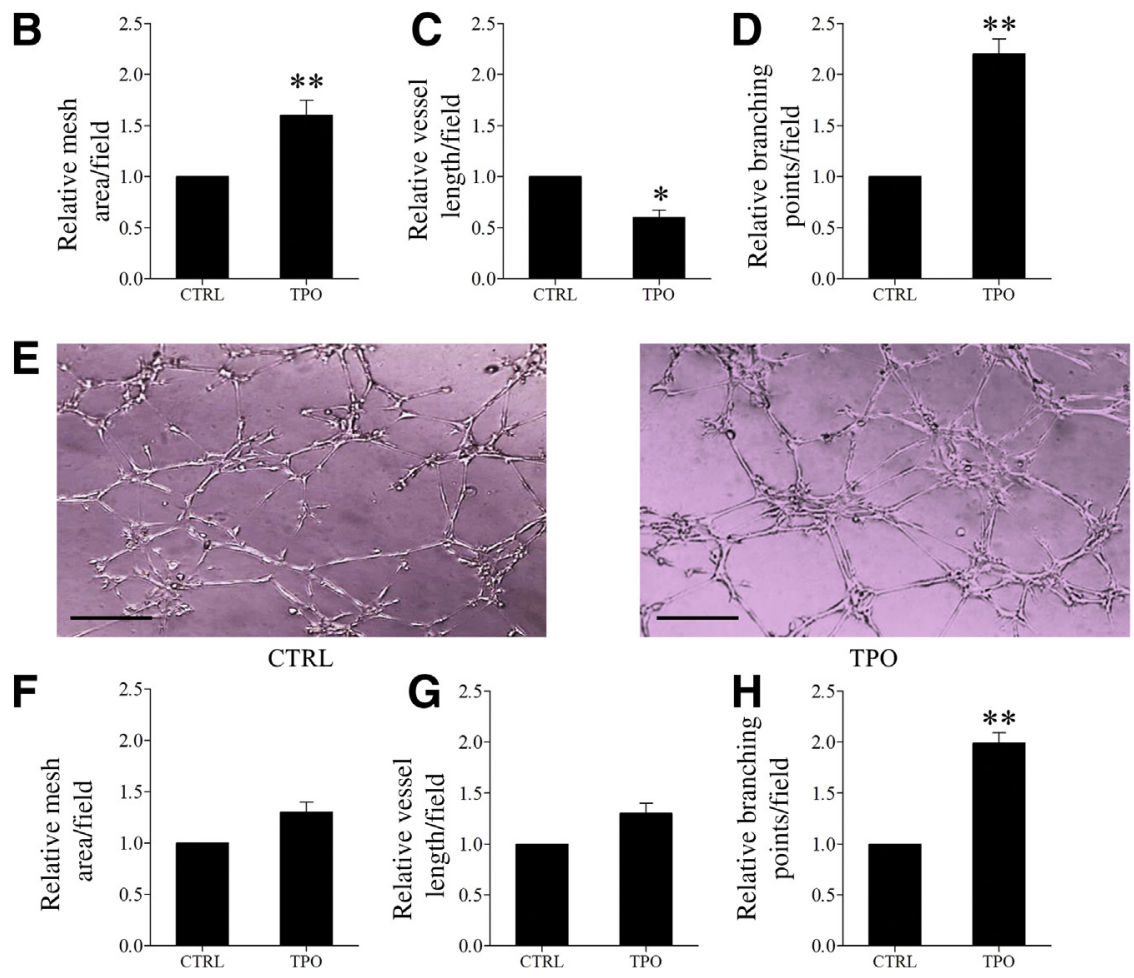

Figure 3 Thrombopoietin (TPO) treatment triggers in vitro Matrigel angiogenesis. Endothelial cells from monoclonal gammopathy of undetermined significance (MGECs; A-D) and endothelial cells from multiple myeloma (MM; MMECS) from first diagnosed $M M(\mathbf{E}-\mathbf{H})$ were treated or not with TPO (10 $\mathrm{ng} / \mathrm{mL})$. Representative images of five independent in vitro angiogenesis assays are shown. Bar graphs represent relative mesh area (B and $\mathbf{F}$ ), vessel length ( $\mathbf{C}$ and $\mathbf{G})$, and branching points ( $\mathbf{D}$ and $\mathbf{H})$, analyzed by EVOS software version 2.0.0 (EuroClone). Note that TPO increases in vitro angiogenesis of MGECS and MMECs. Data are expressed as means \pm SD (B-D and $\mathbf{F}-\mathbf{H})$. ${ }^{*} P<0.05,{ }^{*} P<0.01$ versus control. Scale bars $=50 \mu \mathrm{m}(\mathbf{A}$ and $\mathbf{E})$. Original magnification, $\times 200$ (A and E). CTRL, control.
The angiogenic ability of TPO was confirmed in vivo in the CAM assay, where the sponge was loaded with TPO. Significantly higher newly formed capillaries, spreading radially toward the sponge (mean number of vessels around the implant, $26 \pm 4$ ), compared with negative control (mean number of vessels, $8 \pm 2$ ) (Figure 5), were observed in ovo.

Overall, these results support the angiogenic activity of TPO and suggest that it may have a critical role in promoting the angiogenic switch during MM progression.

\section{TPO Regulates MMP and Cytokine Secretion}

To investigate whether the TPO-induced migration of BMECs occurs through the modulation of matrix metalloproteinases (MMPs) release, the secretion of MMP-9 and MMP-2, the most important MMPs involved in the MM angiogenesis, ${ }^{17}$ was evaluated by the zymography assay. TPO significantly increased the secretion and the activation of MMP-9 and MMP-2 in MGECs (Figure 6, A and C) and the activation of MMP-2 in MMECs (Figure 6, B and D).
Next, the effect of TPO on cytokine secretion by BMECs was evaluated using the human angiogenesis array on conditioned media of MGECs and MMECs, treated or not, with TPO. MGECs and MMECs in basal condition showed a different secretion profile of angiogenic cytokines because of MMEC autocrine and paracrine angiogenic loops. ${ }^{7,18,19}$ TPO treatment significantly increased the secretion of the angiogenic factors angiopoietin-1, insulin-like growth factor-binding protein-2, phosphatidylinositol-glycan biosynthesis class F protein (PIGF), and VEGF by MGECs and monocyte chemoattractant protein-1 and VEGF by MMECs (Figure 7, A and B). By contrast, the antiangiogenic cytokines pentraxin 3 (PTX3) and tissue inhibitors of metalloproteinase-1 (TIMP-1) decreased in MGECs. Furthermore, there was a trend in TPO-mediated angiogenic function modulation (Figure 7, C and D), thus altering the balance between angiogenic/antiangiogenic factors.

Overall, these results suggest that TPO affects MM angiogenesis by regulating the secretion of MMPs and angiogenic cytokines. 
A

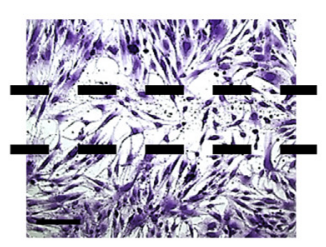

SFM

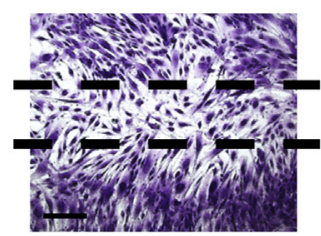

VEGF/FGF-2

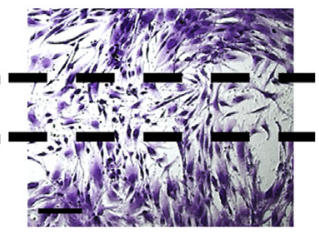

TPO
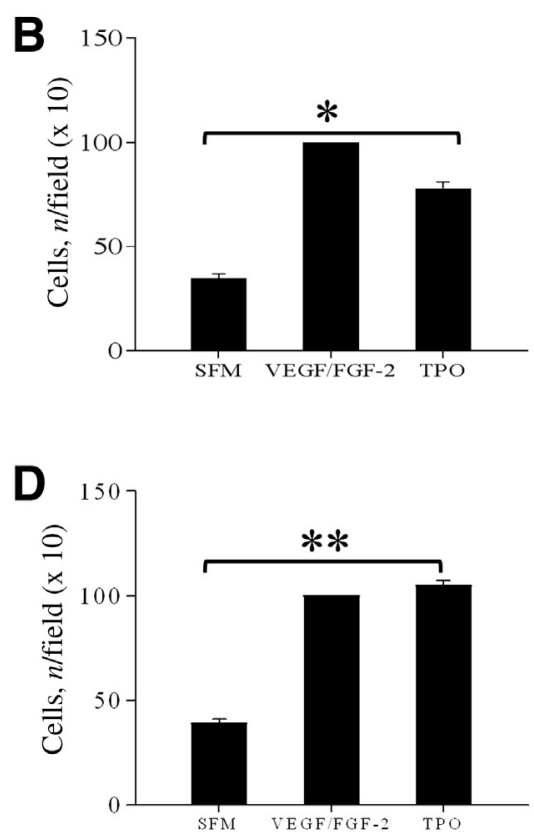

TPO

E

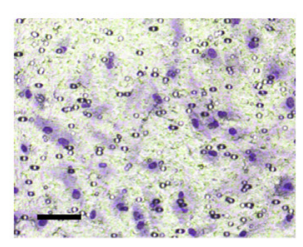

SFM

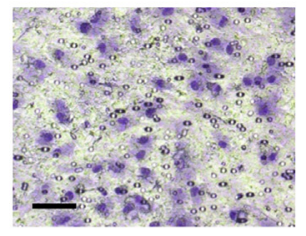

VEGF/FGF-2

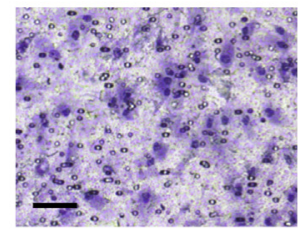

TPO
F

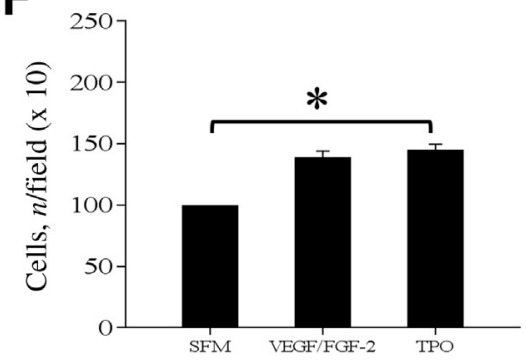

H

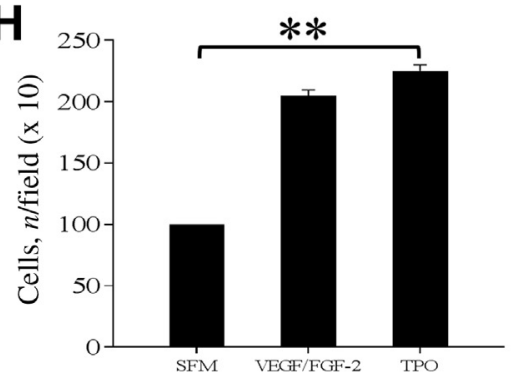

Figure 4 Thrombopoietin (TP0) promotes bone marrow-derived endothelial cell migration. A-D: Spontaneous migration in the wound-healing assay. Endothelial cells from monoclonal gammopathy of undetermined significance (MGECs) and endothelial cells from multiple myeloma (MMECs) from patients at different progression phases were treated with TPO $(10 \mathrm{ng} / \mathrm{mL}$ ) or vascular endothelial growth factor (VEGF)/fibroblast growth factor (FGF)-2 (positive control). A-D: Representative images of five independent wound closures, highlighted by dashed lines (A and C), 16 hours after the scratch of MGECs (A and B) and MMECs from first diagnosed (FIR) patients ( $C$ and $\mathbf{D}$ ), are shown. Bar graphs represent migrated cells per field. E-H: MGECs and MMECs from patients at different progression phases were tested for chemotaxis toward chemotactic medium as positive control (VEGF/FGF-2) and toward TPO. E-H: Representative images of five independent experiments of migrated MGECs (E and $\mathbf{F})$ and MMECs from FIR patients $(\mathbf{G}$ and $\mathbf{H})$ are shown. Bar graphs represent migrated cells per field. Data are expressed as means \pm SD (B, D, F, and $\mathbf{H}) .{ }^{*} P<0.05,{ }^{*} P<0.01$. Scale bars $=50 \mu \mathrm{m}(\mathbf{A}, \mathbf{C}, \mathbf{E}$, and $\mathbf{G})$. Original magnification, $\times 200(\mathbf{A}, \mathbf{C}$, E, and G). bFGF, basic FGF; SFM, serum-free medium.

\section{Discussion}

The transition from MGUS to MM is accompanied by the angiogenic switch that implies the acquisition of angiogenic properties by quiescent ECs and tumor vascularization. Several studies clearly demonstrate the important role of angiogenesis in $\mathrm{MM}$ tumor progression and drug resistance. ${ }^{16,20}$ 

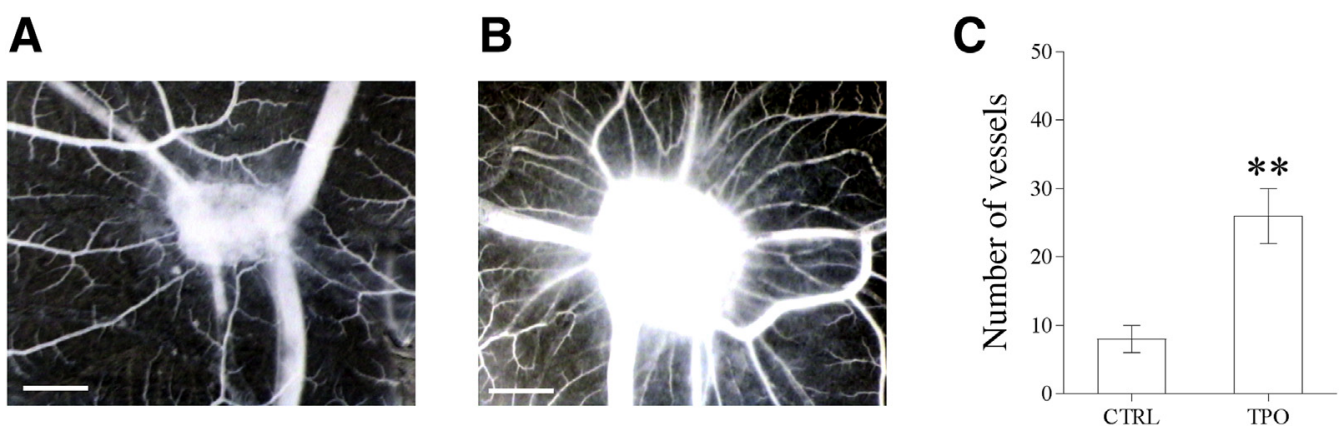

Figure 5 Thrombopoietin (TPO) triggers in vivo angiogenesis. A and B: Chorioallantoic membrane shows vessel formation toward the sponge induced by TPO $(100 \mathrm{ng} / \mathrm{mL} ; \mathbf{B})$ versus negative control (CTRL; A). Representative images on a stereomicroscope. C: Bar graph represents data expressed from three independent experiments. Data are expressed as means \pm SD (C). ${ }^{* * P}<0.01$ versus control. Scale bars $=160 \mu \mathrm{m}(\mathbf{A}$ and $\mathbf{B})$. Original magnification, $\times 50(\mathbf{A}$ and $\mathbf{B})$.

Conventional and high-dose chemotherapies, and/or MM plasma cells infiltration within BM, along with disease progression induce thrombocytopenia that increase TPO levels in bloodstream to ensure platelet production. ${ }^{9}$ Thrombocytopenia is considered a negative predictive and prognostic factor in MM patients. As TPO levels negatively correlate to platelet counts, TPO increase parallels thrombocytopenia. ${ }^{21}$ Indeed, TPO raises in accordance with a reduction of platelet count during the MM progression. High TPO levels not only support thrombopoiesis, but also may have systemic effects that promote MM progression. ${ }^{13,22}$ Ancillary cell communication that occurs among the MM niche at the cellular level actively involves TPO and other cytokines that have interactions among themselves, promoting activation of several cellular pathways (ie, Janus kinase/STAT, RAS/RAF and mitogen-activated protein kinase, Akt, and mTOR pathways). ${ }^{14}$ Such a pathophysiological connection suggests that cytokines are biologically connected, especially while considering oxidative stress within the neoplastic evolution and under therapeutic pressure. $^{23}$ Within the MM environment, inflammation and oxidation shape BM microenvironment suitable for growth.
In this study, we demonstrate that TPO levels correlate with MM progression, and that TPO acts as an angiogenic factor. Indeed, BM/peripheral plasma TPO levels parallel disease progression from MGUS to MM and/or from FIR to REC/DR MM, suggesting that it may represent an unfavorable biomarker of MM patients.

Brizzi et $\mathrm{al}^{13}$ demonstrated that TPO induces an angiogenic response in HUVECs. Herein, TPO triggers the angiogenic functions of BMECs from patients at different progression phases, including MGUS, SMM, FIR MM, relapsed MM, and DR MM. Of particular note, BMECs from patients at different progression phases express similar levels of TPO receptor c-Mpl. This suggests that TPOdependent angiogenesis is related to TPO levels and to the activation of TPO/c-Mpl receptor pathway. TPO favors BMECs spontaneous migration and acts as chemoattractant factor, inducing BMECs motility. Indeed, TPO-treated BMECs were able to close the wound in the woundhealing assay more than untreated BMECs. Romanelli et $\mathrm{al}^{24}$ asserted that the activation of TPO/c-Mpl pathway on hepatoblastoma cells was associated with biological activities relevant for tumor progression. In particular, TPOtreated HepG2 cells showed increased migration and
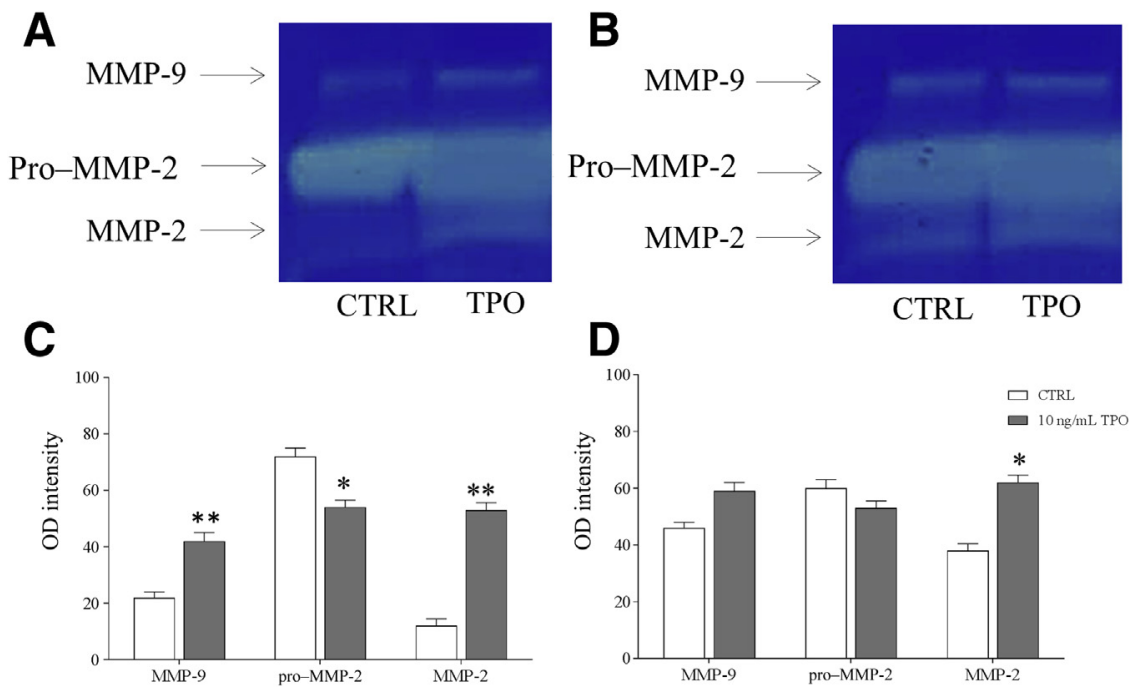

Figure 6 Thrombopoietin (TP0) increases matrix metalloproteinases (MMPs) secretion and activation in bone marrow-derived endothelial cells. Conditioned media of endothelial cells from monoclonal gammopathy of undetermined significance (MGECs) and endothelial cells from multiple myeloma (MMECs) from patients at different progression phases cultured in absence or presence of TPO $(10 \mathrm{ng} / \mathrm{mL})$ were tested for zymography to determine the amount of MMP-2 and MMP-9. Region of protease activity appeared as white bands, and the percentage of MMPs release was estimated using Gel Logic 1500 Imaging System. Representative images of three independent experiments of MGECs ( $\mathbf{A}$ and $\mathbf{C}$ ) and MMECs from first diagnosed patients (B and $\mathbf{D})$ are shown. Note that TPO treatment promotes the secretion of MMPs. Data are expressed as means $\pm \mathrm{SD}(\mathbf{C}$ and $\mathbf{D})$. ${ }^{*} P<0.05$, $* * P<0.01$ versus control. CTRL, control. 
A
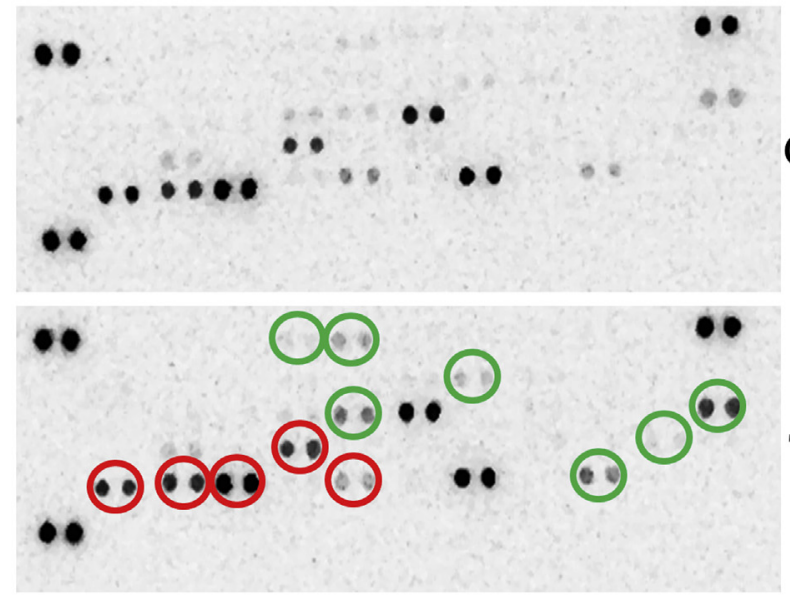

PROANGIOGENIC
Angiogenin

Angiopoietin-1*

Endothelin-1

IGFBP-2*

MCP-1

PIGF*

VEGF**
ANTIANGIOGENIC

PTX3*

PAI-1

Serpin-F1

TIMP-1*

Thrombospondin-1
B
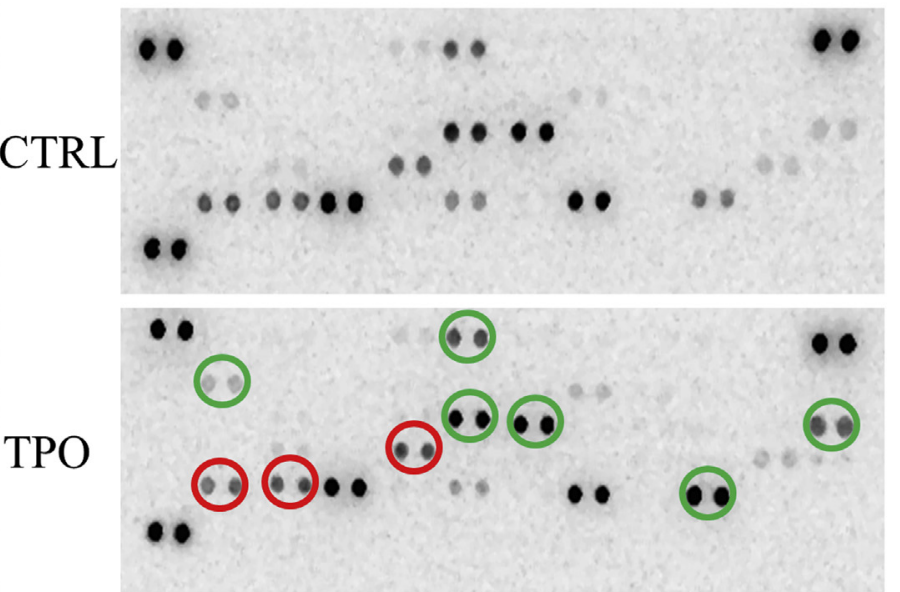

C

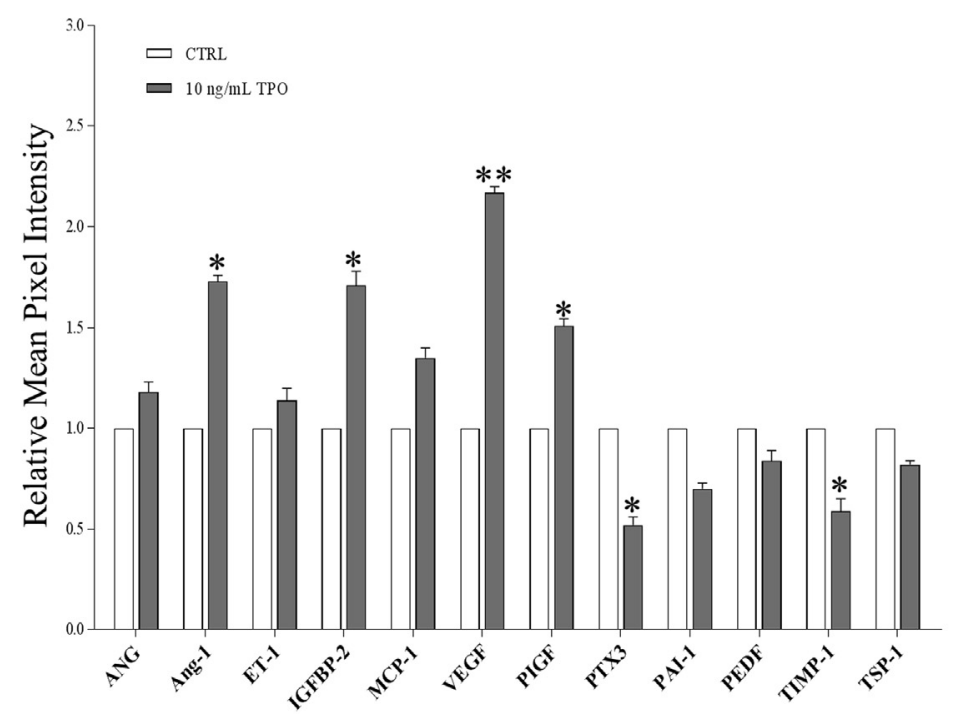

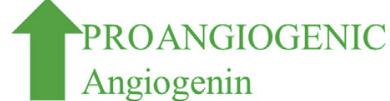

Angiogenin

CXCL16

IGFBP-2/IGFBP-3

MCP-1**

$\mathrm{VEGF}^{* *}$

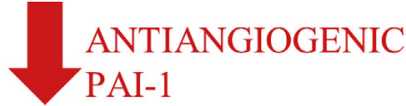

Thrombospondin-1

D

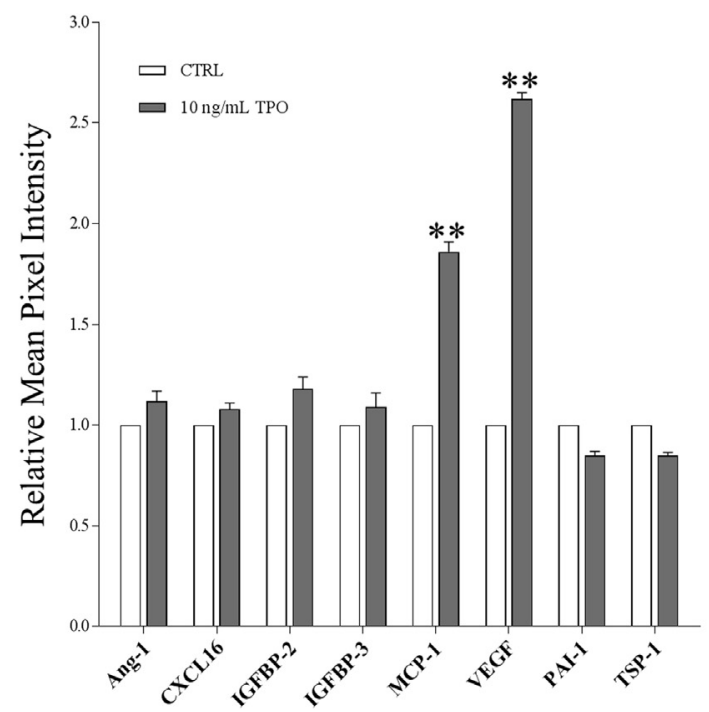

Figure 7 Thrombopoietin (TP0) regulates secretion of cytokines involved in angiogenesis. Conditioned media of endothelial cells from monoclonal gammopathy of undetermined significance (MGECs) and endothelial cells from multiple myeloma (MMECs) from patients at different progression phases cultured in absence or presence of TPO $(10 \mathrm{ng} / \mathrm{mL})$ were analyzed for angiogenic cytokine/growth factor secretion by the angiogenesis assay. A and B: Representative images of three independent experiments of MGECS (A) and MMECs (B) from first diagnosed patients are shown. C and D: Expression of overall data. Note that TPO treatment modulates proangiogenic/antiangiogenic cytokines release, supporting the angiogenic process. Data are expressed as means $\pm \mathrm{SD}$ (C and $\mathbf{D})$. ${ }^{*} P<0.05,{ }^{*} P<<0.01$ versus control. ANG, angiogenin; Ang-1, angiopoietin-1; CTRL, control; ET-1, endothelin-1; IGFBP, insulin-like growth factor-binding protein; MCP-1, monocyte chemoattractant protein-1; PAI-1, serpin E1; PEDF, serpin F1; PIGF, phosphatidylinositolglycan biosynthesis class F protein; PTX3, pentraxin 3; TIMP-1, tissue inhibitors of metalloproteinase-1; TSP-1, thrombospondin-1; VEGF, vascular endothelial growth factor. 
chemoinvasion that sustained the in vivo diffusion of hepatoblastoma within the liver parenchyma. TPO acts as chemoattractant factor on BMECs, triggering BMECs motility in chemotaxis assay. Segerer et $\mathrm{al}^{25}$ studied the ability of TPO to function as a chemokine on decidual cells. Interestingly, they found that TPO stimulated the migratory activity of immune cells, including natural killer cells and $\mathrm{CD} 14^{+}$monocytes, and of cytotrophoblast cells during early gestation. Therefore, TPO has chemotactic and chemokinetic effects and supports the placentation process by promoting the migration of decidual subset cells and their proliferation and cytokine secretion. Brizzi et $\mathrm{al}^{13}$ demonstrated that TPO increases human umbilical vein EC migration and angiogenic response through the phosphorylation of STAT proteins.

Current results show that TPO triggers angiogenesis in the in vitro and in vivo CAM assay. Indeed, the formation of meshwork of capillary-like structures on Matrigel is elicited by TPO, particularly in MGECs, whose ability to form meshes is minimal in untreated cells; in MMECs, TPO further increases the in vitro angiogenic network. TPO has a greater angiogenic effect on MGECs because MMECs already have constitutive angiogenic ability. ${ }^{26}$ Furthermore, for the first time, we demonstrate the effect of TPO in ovo in CAM assay, confirming the angiogenic role of TPO in vivo in a mouse model of Matrigel implantation. ${ }^{13}$

Remarkably, BMECs from patients at different progression phases express similar levels of the TPO receptor c-Mpl. This suggests that TPO-dependent angiogenesis is related to TPO levels. Furthermore, c-Mpl/TPO pathway regulates the secretion of cytokines and MMPs involved in angiogenic process. MMPs are implied in breakdown of extracellular matrix by proteolytic degradation necessary for new vessel formation. They also promote tumor progression, invasion, metastasis, and angiogenesis by regulating ECs attachment, proliferation, migration, and growth, either directly or by release of sequestered growth factors. ${ }^{27}$ MMP-2 is closely involved in ECs migration, affecting extracellular matrix turnover and modifying cell-extracellular matrix interactions that result in the angiogenic response. MM angiogenesis depends on MMP-2: ECs from active MM patients express higher levels of MMP2 compared with MGECs, whereas MMP-9 expression was similar in all patient groups. ${ }^{28}$ Accordingly, here, TPO significantly activated both MMP-9 and MMP-2 in MGECs and MMP-2 in MMECs, thus further increasing the higher MMP-2 levels of MMECs.

Several growth factors act via autocrine and paracrine mechanisms, modulating angiogenesis. ${ }^{3}$ In particular, autocrine/paracrine loops of angiogenic cytokines are involved in MM tumor progression and angiogenesis [ie, hepatocyte growth factor (HGF)/tyrosine-protein kinase Met (c-MET), epidermal growth factor (EGF), and VEGF loops, the angiogenic Epo, and the anti-angiogenic PTX3]. ${ }^{7,18,19,29,30}$ Accordingly, herein TPO modulated the BM angiogenic milieu by increasing angiogenic cytokines (angiopoietin-1, insulin-like growth factor-binding protein-2, PIGF, VEGF, and monocyte chemoattractant protein-1) and by decreasing the antiangiogenic ones (PTX3 and TIMP-1). Overall, our results highlight a relationship between $\mathrm{BM} /$ peripheral plasma TPO levels and disease progression in accordance to thrombocytopenia and BM angiogenesis. Therefore, TPO may be envisaged as a novel angiogenic and prognostic factor in MM.

\section{Author Contributions}

A.L. conceived and supervised the study; A.L. and I.S. designed the experiments; A.L., I.S., D.R., V.D., and M.A.F. performed the experiments; A.L., I.S., and M.A.F. analyzed the data and prepared the manuscript; A.G.S., A.M., R.R., and A.V. revised the manuscript; all authors approved the published version of the manuscript.

\section{Supplemental Data}

Supplemental material for this article can be found at http://doi.org/10.1016/j.ajpath.2020.12.016.

\section{References}

1. Palumbo A, Anderson K: Multiple myeloma. N Engl J Med 2011, 364 : 1046-1060

2. Di Marzo L, Desantis V, Solimando AG, Ruggieri S, Annese T, Nico B, Fumarulo R, Vacca A, Frassanito MA: Microenvironment drug resistance in multiple myeloma: emerging new players. Oncotarget 2016, 7:60698-60711

3. Vacca A, Ribatti D: Bone marrow angiogenesis in multiple myeloma. Leukemia 2006, 20:193-199

4. Roccaro AM, Hideshima T, Raje N, Kumar S, Ishitsuka K, Yasui H, Shiraishi N, Ribatti D, Nico B, Vacca A, Dammacco F, Richardson PG, Anderson KC: Bortezomib mediates antiangiogenesis in multiple myeloma via direct and indirect effects on endothelial cells. Cancer Res 2006, 66:184-191

5. De Luisi A, Ferrucci A, Coluccia AM, Ria R, Moschetta M, de Luca E, Pieroni L, Maffia M, Urbani A, Di Pietro G, Guarini A, Ranieri G, Ditonno P, Berardi S, Caivano A, Basile A, Cascavilla N, Capalbo S, Quarta G, Dammacco F, Ribatti D, Vacca A: Lenalidomide restrains motility and overangiogenic potential of bone marrow endothelial cells in patients with active multiple myeloma. Clin Cancer Res 2011, 17: 1935-1946

6. Ria R, Catacchio I, Berardi S, De Luisi A, Caivano A, Piccoli C, Ruggieri V, Frassanito MA, Ribatti D, Nico B, Annese T, Ruggieri S, Guarini A, Minoia C, Ditonno P, Angelucci E, Derudas D, Moschetta M, Dammacco F, Vacca A: HIF-1 $\alpha$ of bone marrow endothelial cells implies relapse and drug resistance in patients with multiple myeloma and may act as a therapeutic target. Clin Cancer Res 2014, 20:847-858

7. Ferrucci A, Moschetta M, Frassanito MA, Berardi S, Catacchio I, Ria R, Racanelli V, Caivano A, Solimando AG, Vergara D, Maffia M, Latorre D, Rizzello A, Zito A, Ditonno P, Maiorano E, Ribatti D, Vacca A: A HGF/cMET autocrine loop is operative in multiple myeloma bone marrow endothelial cells and may represent a novel therapeutic target. Clin Cancer Res 2014, 20:5796-5807

8. Albarracin F, Fonseca R: Plasma cell leukemia. Blood Rev 2011, 25: $107-112$

9. Folman CC, de Jong CM, de Haas M, von dem Borne AE: In multiple myeloma increased thrombopoietin (Tpo) production may be involved 
in the maintenance of platelet production. Eur J Haematol 2001, 66: $337-341$

10. Wendling F, Maraskovsky E, Debili N, Florindo C, Teepe M, Titeux M, Methia N, Breton-Gorius J, Cosman D, Vainchenker W: $\mathrm{cMpl}$ ligand is a humoral regulator of megakaryocytopoiesis. Nature 1994, 369:571-574

11. Hitchcock IS, Kaushansky K: Thrombopoietin from beginning to end. Br J Haematol 2014, 165:259-268

12. Sungaran R, Markovic B, Chong BH: Localization and regulation of thrombopoietin $\mathrm{mRNa}$ expression in human kidney, liver, bone marrow, and spleen using in situ hybridization. Blood 1997, 89: $101-107$

13. Brizzi MF, Battaglia E, Montrucchio G, Dentelli P, Del Sorbo L, Garbarino G, Pegoraro L, Camussi G: Thrombopoietin stimulates endothelial cell motility and neoangiogenesis by a platelet-activating factor-dependent mechanism. Circ Res 1999, 84:785-796

14. Kuter DJ: The biology of thrombopoietin and thrombopoietin receptor agonists. Int J Hematol 2013, 98:10-23

15. Rajkumar SV, Dimopoulos MA, Palumbo A, Blade J, Merlini G, Mateos MV, Kumar S, Hillengass J, Kastritis E, Richardson P, Landgren O, Paiva B, Dispenzieri A, Weiss B, LeLeu X, Zweegman S, Lonial S, Rosinol L, Zamagni E, Jagannath S, Sezer O, Kristinsson SY, Caers J, Usmani SZ, Lahuerta JJ, Johnsen HE, Beksac M, Cavo M, Goldschmidt H, Terpos E, Kyle RA, Anderson KC, Durie BG, Miguel JF: International Myeloma Working Group updated criteria for the diagnosis of multiple myeloma. Lancet Oncol 2014, 15:e538-e548

16. Ria R, Berardi S, Reale A, Luisi AD, Catacchio I, Racanelli V, Vacca A: Multiple myeloma: the role of angiogenesis in disease progression. J Bone Marrow Res 2013, 1:117

17. Vacca A, Ribatti D, Roccaro AM, Ria R, Palermo L, Dammacco F: Bone marrow angiogenesis and plasma cell angiogenic and invasive potential in patients with active multiple myeloma. Acta Haematol 2001, 106:162-169

18. Rao L, Giannico D, Leone P, Solimando AG, Maiorano E, Caporusso C, Duda L, Tamma R, Mallamaci R, Susca N, Buonavoglia A, Da Vià MC, Ribatti D, De Re V, Vacca A, Racanelli V: HB-EGF-EGFR signaling in bone marrow endothelial cells mediates angiogenesis associated with multiple myeloma. Cancers $2020,12: 173$

19. Vacca A, Ria R, Ribatti D, Semeraro F, Djonov V, Di Raimondo F, Dammacco F: A paracrine loop in the vascular endothelial growth factor pathway triggers tumor angiogenesis and growth in multiple myeloma. Haematologica 2003, 88: $176-185$
20. Fan F, Schimming A, Jaeger D, Podar K: Targeting the tumor microenvironment: focus on angiogenesis. J Oncol 2012, 2012:281261

21. Mellors PW, Binder M, Buadi FK, Lacy MQ, Gertz MA, Dispenzieri A, Hayman SR, Kapoor P, Gonsalves WI, Hwa YL, Fonder A, Hobbs M, Kourelis T, Warsame R, Zeldenrust SR, Lust JA, Leung N, Go RS, Kyle RA, Rajkumar SV, Kumar SK: Development of thrombocytopenia during first-line treatment and survival outcomes in newly diagnosed multiple myeloma. Leuk Lymphoma 2019, 60: 2960-2967

22. Kamińska J, Koper OM, Mantur M, Matowicka-Karna J, SawickaPowierza J, Sokołowski J, Kostur A, Kulczyńska A, Kłoczko J, Kemona H: Does thrombopoiesis in multiple myeloma patients depend on the stage of the disease? Adv Med Sci 2014, 59:166-171

23. Zhang J, Lei W, Chen X, Wang S, Qian W: Oxidative stress response induced by chemotherapy in leukemia treatment. Mol Clin Oncol 2018, 8:391-399

24. Romanelli RG, Petrai I, Robino G, Efsen E, Novo E, Bonacchi A, Pagliai G, Grossi A, Parola M, Navari N, Delogu W, Vizzutti F, Rombouts K, Gentilini P, Laffi G, Marra F: Thrombopoietin stimulates migration and activates multiple signaling pathways in hepatoblastoma cells. Am J Physiol Gastrointest Liver Physiol 2006, 290:G120-G128

25. Segerer SE, Martignoni F, Bogdan A, Müller N, Kapp M, Dietl J, Rieger L, Kämmerer U: Thrombopoietin modulates the proliferation, migration and cytokine profile of decidual cell subsets during early gestation. Mol Hum Reprod 2013, 19:361-368

26. Ria R, Reale A, De Luisi A, Ferrucci A, Moschetta M, Vacca A: Bone marrow angiogenesis and progression in multiple myeloma. Am J Blood Res 2011, 1:76-89

27. Kalluri R: Basement membranes: structure, assembly and role in tumour angiogenesis. Nat Rev Cancer 2003, 3:422-433

28. Vacca A, Ribatti D, Presta M, Minischetti M, Iurlaro M, Ria R, Albini A, Bussolino F, Dammacco F: Bone marrow neovascularization, plasma cell angiogenic potential, and matrix metalloproteinase-2 secretion parallel progression of human multiple myeloma. Blood 1999, 93:3064-3073

29. Lamanuzzi A, Saltarella I, Ferrucci A, Ria R, Ruggieri S, Racanelli V, Rao L, Annese T, Nico B, Vacca A, Ribatti D: Role of erythropoietin in the angiogenic activity of bone marrow endothelial cells of MGUS and multiple myeloma patients. Oncotarget 2016, 7:14510-14521

30. Basile A, Moschetta M, Ditonno P, Ria R, Marech I, De Luisi A, Berardi S, Frassanito MA, Angelucci E, Derudas D, Specchia G, Curci P, Pavone V, Rossini B, Ribatti D, Bottazzi B, Mantovani A, Presta M, Dammacco F, Vacca A: Pentraxin 3 (PTX3) inhibits plasma cell/stromal cell cross-talk in the bone marrow of multiple myeloma patients. J Pathol 2013, 229:87-98 